\title{
A Case of Angioleiomyoma of the Maxillary Sinus
}

\section{Ick Soo Choi}

Department of Otorhinolaryngology, College of Medicine, Inje University, Ilsan Paik Hospital, Koyang, Korea

Email: leochoics@gmail.com

How to cite this paper: Choi, I.S. (2019) A Case of Angioleiomyoma of the Maxillary Sinus. International Journal of Otolaryngology and Head \& Neck Surgery, 8, 7-12. https://doi.org/10.4236/ijohns.2019.81002

Received: October 27, 2018

Accepted: December 7, 2018

Published: December 10, 2018

Copyright $\odot 2019$ by author and Scientific Research Publishing Inc. This work is licensed under the Creative Commons Attribution International License (CC BY 4.0).

http://creativecommons.org/licenses/by/4.0/

\section{Open Access}

\begin{abstract}
Angioleiomyoma (ALM) is a benign tumor that occurs most frequently in the subcutaneous tissue of the upper and lower extremities. ALM arising in the sinonasal tract is rare, with fewer than $1 \%$ of cases presenting in this region. Only two cases localized to the sinuses have been reported to date in the literature. We report a case of a 30-year-old man complaining of a headache noted to have a concomitant lesion in the maxillary sinus. Histopathological examination following surgical resection confirmed a diagnosis of ALM. This report is the first known report of ALM limited to the maxillary sinus, and the first report to present the imaging data in the ALM in the sinus.
\end{abstract}

\section{Keywords}

Angioleiomyoma, Maxillary Sinus

\section{Introduction}

Angioleiomyoma (ALM; synonyms: angiomyoma, vascular leiomyoma) is a well-differentiated, benign smooth muscle tumor often associated with a vascular component. It occurs in the dermis and subcutaneous tissue of the limbs most frequently, presenting as a painful slowly enlarging nodule in 30 - 50 years old patients [1]. Although the lower limb is the most common site in which ALM is diagnosed, prevalence of the tumor in the head and neck is reported to be between $8.5 \%$ - 13\%. However, reports of ALM being limited to the sinonasal tract, an uncommon site for parenchymal tumors, is infrequent, with fewer than $1 \%$ of cases localized to this region [2] [3]. Further, only two clinical reports of ALM limited to the paranasal sinuses have been published to date [2] [4].

We report a case of a 30-year-old male presenting with headache found to have histopathologically confirmed ALM of the maxillary sinus. We believe this to be the first report of ALM confined to the maxillary sinus. 


\section{Case Report}

A 30-year-old man with no past medical history presented to our neurology department with a 5-year history of headache. He was referred to our clinic following the discovery of a left maxillary sinus mass on magnetic resonance imaging (MRI) of the brain. The patient denied all nasal symptoms including rhinorrhea, nasal obstruction, and postnasal drip. Nasal endoscopy demonstrated edema of the left middle meatal mucosa, but did not reveal the presence of a mass.

T1-weighted (T1WI) MRI showed a lesion in the left maxillary sinus isointense with respect to skeletal muscle. T2-weighted images (T2WI) showed a corresponding heterogeneous, hyperintense lesion relative to muscle (Figure 1).

Paranasal sinus (PNS) computed tomography (CT) showed an expansile, peripherally enhancing lesion occupying the left maxillary sinus. The lesion extended into the middle nasal meatus through the natural ostium. Some calcification of the maxillary sinus antrum was observed without evidence of bony destruction (Figure 2).

Our presumptive pre-operative diagnosis was inverted papilloma, the most common benign neoplasm of the PNS. The patient underwent Caldwell-Luc surgery combined endoscopic sinus surgery under general anesthesia. Intraoperatively, mass was found to be adhered to the uncinate process. The tumor was more adherent to surrounding tissues than typically observed with inverted papilloma. A clearly differentiated origin could not be appreciated. The excised reddish-pink mass measured $3.5 \times 3.2 \mathrm{~cm}$. Minimal blood loss occurred during surgery.

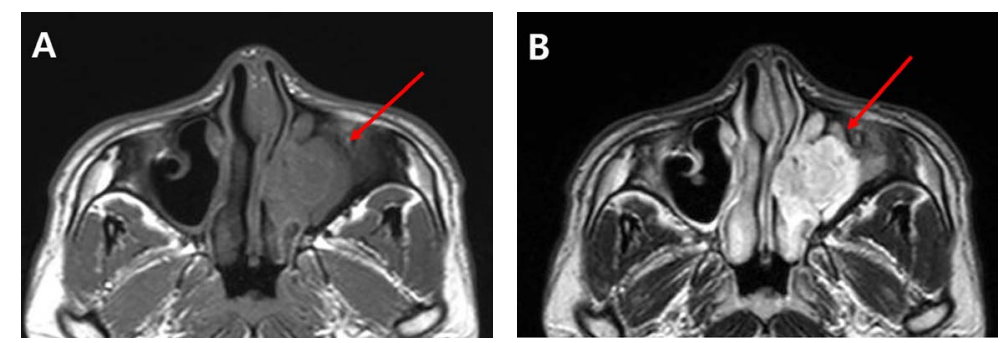

Figure 1. Pre-operative brain MRI. (A) T1-weighted image showing the lesion located to the left maxillary sinus is isointense with surrounding muscle tissue; (B) T2-weighted image showing a hyperintense lesion in the left maxillary sinus.

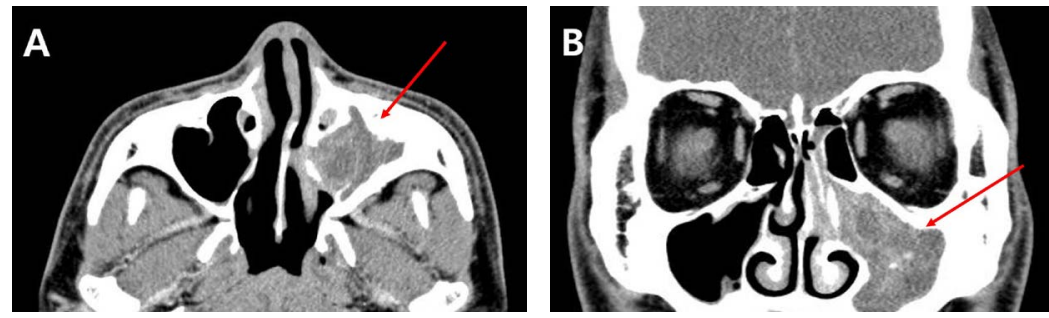

Figure 2. Pre-operative paranasal Sinus CT. (A) Axial view shows an expansile, peripheral enhancing lesion occupying the left maxillary sinus with bulging into the middle nasal meatus through the natural ostium; (B) Coronal view shows the same lesion. 
Histopathological examination of the tissue was performed. Hematoxylin and eosin ( $\mathrm{H} \& \mathrm{E})$ stain of the specimen showed numerous thick and thin-walled vascular structures within the tumor. The vascular wall was composed of poorly-differentiated, spindle-shaped smooth muscle cells with minimal nuclear atypia. The vessels within the tumor were difficult to identify histopathologically due to the flattening of the lumen, and the spindle cells of the vessel wall were less differentiated than the original soft tissue vascular leiomyoma (Figure 3). Due to difficulty in identifying intratumoral vasculature, we performed immunohistochemical staining for smooth muscle actin (SMA). This allowed us to differentiate ALM with other vascular tumors such as angiofibroma or extrapleural solitary fibrous tumor. The tumor displayed a diffuse, strongly positive response to SMA stain, yielding a final diagnosis of vascular leiomyoma (Figure $4)$.

Following surgery, the patient experienced complete resolution of symptoms. Twenty-four-month follow up was uncomplicated, without complications or tumor recurrence.

The patient has given his consent for the publication of his case and his images.

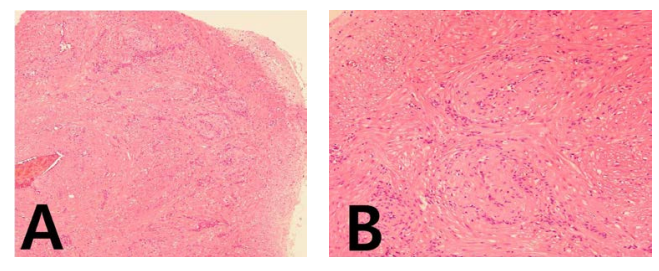

Figure 3. Hematoxalin and Eosin ( $\mathrm{H} \& \mathrm{E}$ ) Immunohistochemical Stain. (A) Original magnification $\times 40$; $(B)$ Original magnification $\times 100$. Demonstrated well-demarcated, smooth muscle tissue with intratumoral thick and thin-walled vessels, low cellularity, no mitosis.

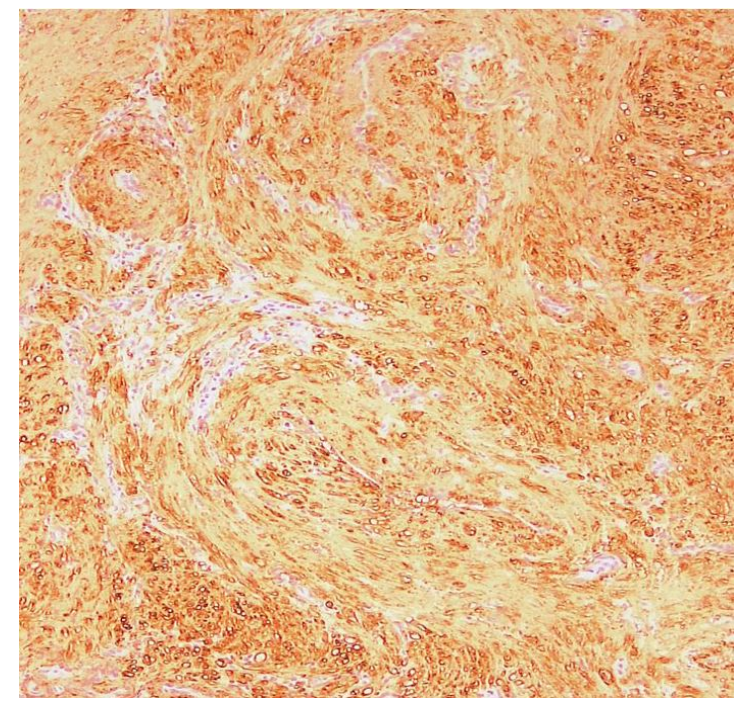

Figure 4. Smooth Muscle Actin (SMA) Stain. This immunohistochemical stain revealed a positive reaction to the actin filament of smooth muscle fibers. 


\section{Discussion}

The World Health Organization classifies ALM as a type of pericystic (perivascular) tumor [1]. Previously, it had been regarded as a smooth muscle tumor along with deep and genital leiomyoma [5].

ALM is most commonly seen in the lower extremities, particularly amongst women between 30 and 50 years of age [2]. There have also been reports of ALM presentations in the dermis overlying the neck, ear, external nose, forehead, lips, and internal can thus. However, ALM has rarely been implicated in the sinonasal tract. In a retrospective review of 21 cases of head and neck ALM, Wang et al. reported only two tumors affecting the sinonasal tract [3]. Similarly, of 12 patients diagnosed with ALM, Yoon et al. reported five neoplasms within the sinonasal tract [6]. These cases mainly affected the nasal turbinates, septum, and vestibule, with only two localized to the paranasal sinuses.

ALM is typically characterized as a slow growing, firm, mobile, and solitary subcutaneous mass measuring less than $2 \mathrm{~cm}$. Patients most often complain of pain in the extremities. Sinonasal ALM commonly presents with epistaxis and nasal obstruction. Less often reported symptomology may include facial pain, headache, and epiphora.

Radiologic features of ALM are most effectively viewed using MRI. T1WI show isointense signal, while T2WI show mixed areas of hyper and isointensity relative to skeletal muscle. Also seen in the T2WI is a hypointense rim representing the tumor wall. The hyperintense area shows strong enhancement but isointense area by contrast [7].

Of the two reported cases of PNS ALM, neither included clear radiological data. One case detailed a $0.2 \mathrm{~mm}$ microscopic lesion found in a patient with recurrent sinusitis, rendering the imaging inconsequential. The second case described a patient with nasal obstruction and headache resulting from extensive masses in the maxillary sinus, sphenoid sinus, and nasal cavity. However, Schwartzman and Schwartzman published this report in 1973 without endoscopic findings or imaging [4]. Our patient presented with a chief complaint of headache. In this report, we provide MRI findings similar to those described in ALM of the extremities.

Trauma, venous stasis, and hormonal (i.e., estrogenic) changes have been proposed to be causative factors of ALM [7]. In the present case, we were unable to determine a causative factor; however, the patient may have experienced an unreported facial trauma ultimately leading to ALM formation in the maxillary sinus.

Histopathologically, ALM is composed of well-differentiated smooth muscle cells with intervening vascular channels without mitosis. There are three histological classifications of ALM, namely the solid, venous, and cavernous types. Multiple immunohistochemical stains including, Smooth Muscle Actin (SMA), muscle specific actin (MSA), calponin, and h-caldesmon are utilized to differentiate ALM from other neoplasms with a vascular component. Most commonly, 
ALM seen in the sinonasal tract is of the solid histological subtype, as was present in our case [2]. Interestingly, the two previously described cases of ALM affecting the sinuses were not of the solid type.

Most ALM shows benign progress with rare malignant change. Only two cases of malignant transformation of ALM have been reported in the extremities [8] [9]. Surgical resection is considered to be a curative treatment for ALM [10]. There have been no reported incidences of recurrence following resection of ALM from the sinonasal tract. However, there was a reported case of recurrence of ALM in the oral cavity occurring two months after simple resection of the tumor [11]. Therefore, we believe periodic follow-up after resection is necessary. The patient presented in our study showed no evidence of recurrence in the 2 years following surgical resection.

\section{Conclusion}

Recently, we experienced a patient with maxillary sinus angioleiomyoma (ALM). ALM is a benign, well-differentiated smooth muscle tumor which can be found at various organs. However, ALM in the sinonasal tract has rarely been reported. The patient's ALM was successfully resected without complications or recurrence. Significance of the report is that this is the first known report to present ALM limited to the maxillary sinus with imaging data.

\section{Conflicts of Interest}

The author declares no conflicts of interest regarding the publication of this paper.

\section{References}

[1] Hisaoka, M. and Quase, B. (2013) Angioleiomyoma. In: Fletcher, C.D.M., Bridge, J.A., Hogendoorn, P.C.W. and Mertens, F., Eds., World Health Organization Classification of Tumors of Soft Tissue and Bone, 4th Edition, IARC Press, Lyon, 120-121.

[2] Agaimy, A., Michal, M., Thompson, L.D. and Michal, M. (2015) Angioleiomyoma of the Sinonasal Tract: Analysis of 16 Cases and Review of the Literature. Head and Neck Pathology, 9, 463-473. https://doi.org/10.1007/s12105-015-0636-y

[3] Wang, C.P., Chang, Y.L. and Sheen, T.S. (2004) Vascular Leiomyoma of the Head and Neck. Laryngoscope, 114, 661-665. https://doi.org/10.1097/00005537-200404000-00012

[4] Schwartzman, J. and Schwartzman, J. (1973) Leiomyoangioma of Paranasal Sinuses: Case Report. Laryngoscope, 83, 1856-1858. https://doi.org/10.1002/lary.5540831118

[5] Fanburg-Smith, J.C. and Thompson, L.D.R. (2005) Benign Soft Tissue Tumours. In: Barnes, L., Eveson, J.W., Reichart, P. and Sidransky, D., Eds., World Health Organization Classification of Tumors. Pathology and Genetics of Tumours of Hea and Neck Tumours, IARC Press, Lyon, 46-50.

[6] Yoon, T.M., Yang, H.C., Choi, Y.D., Lee, D.H., Lee, J.K. and Lim, S.C. (2013) Vascular Leiomyoma in the Head and Neck Region: 11 Years Experience in One Institution. Clinical and Experimental Otorhinolaryngology, 6, 171-75. https://doi.org/10.3342/ceo.2013.6.3.171 
[7] Ramesh, P., Annapureddy, S.R., Khan, F. and Sutaria, P.D. (2004) Angioleiomyoma: A Clinical, Pathological and Radiological Review. International Journal of Clinical Practice, 58, 587-591. https://doi.org/10.1111/j.1368-5031.2004.00085.x

[8] Herren, D.B., Zimmermann, A. and Buchler, U. (1995) Vascular Leiomyoma in an Index Finger Undergoing Malignant Transformation. Journal of Hand Surgery (European Volume), 20, 484-487. https://doi.org/10.1016/S0266-7681(05)80158-5

[9] Neviaser, R.J. and Newman, W. (1977) Dermal Angiomyoma of the Upper Extremity. Journal of Hand Surgery, 2, 271-274. https://doi.org/10.1016/S0363-5023(77)80125-1

[10] Hachisuga, T., Hashimoto, H. and Enjoji, M. (1984) Angioleiomyoma. A Clinic Opathologic Reappraisal of 562 Cases. Cancer, 54, 126-130. https://doi.org/10.1002/1097-0142(19840701)54:1<126::AID-CNCR2820540125>3.0 .CO;2-F

[11] Mahima, V.G., Patil, K. and Srikanth, H.S. (2011) Recurrent Oral Angioleiomyoma. Contemporary Clinical Dentistry, 2, 102-105.

https://doi.org/10.4103/0976-237X.83071 